\title{
Un nouveau mécanisme de la spécificité de l'action hormonale relayée par les récepteurs nucléaires
}

Les facteurs de transcription de la famille des récepteurs nucléaires ont pour particularité que leur action transcriptionnelle est, dans une large mesure, conditionnée par la liaison d'un ligand. Ces ligands sont très variés, tant en structure qu'en fonction, et incluent les stéroïdes, les hormones thyroïdiennes, la vitamine $\mathrm{D}$ et des morphogènes, tel l'acide rétinoïque ( $m / s n^{\circ} 3$, vol. 6, p. 307). Nous savons depuis longtemps que l'action de ces ligands ou hormones est très spécifique, que l'on considère le tissu ou le gène-cible. Or, l'étude des récepteurs nucléaires et de leurs mécanismes d'action transcriptionnelle a surtout révélé la grande conservation de ces mécanismes [1] ; il nous reste encore à comprendre comment s'opère la spécificité d'action.

Les récepteurs de la sous-famille constituée des récepteurs de l'acide rétinoïque (RAR), de l'acide 9-cis rétinoïque (RXR), des hormones thyroïdiennes $\left(T_{3} R\right)$ et de la vitamine $D$ (VDR), reconnaissent des séquences composées du même hexanucléotide de base (AGGTCA) [2]. On a proposé qu'un code de reconnaissance, basé principalement sur l'espacement entre deux répétitions de ce motif, rende compte de la spécificité d'action des récepteurs $\left(m / s n^{\circ} 8\right.$, vol. 7 , p. 863). Dans ce modèle, le récepteur RXR participe à la formation d'hétérodimères $\left(m / s n^{\circ} 2\right.$, vol. $\left.6, p .165\right)$ en interagissant avec le récepteur qui détermine la réponse hormonale spécifique, par exemple le $T_{3} R$ pour un gène répondant aux hormones thyroïdiennes ; RXR lie la moitié amont des sites sur l'ADN, alors que la moitié aval est reconnue par $\mathrm{T}_{3} \mathrm{R}$ [1]. Ce modèle a été développé en bonne partie en utilisant des cibles d'ADN synthétiques $\left(m / s n^{\circ} 3\right.$, vol. 4, p. 196). Or, un bon nombre des cibles d'ADN naturelles que l'on retrouve dans les promoteurs de gènes cibles, ne correspondent pas à ces séquences. Il est donc vraisemblable que d'autres mé- canismes contribuent à la spécificité de la reconnaissance des cibles ADN et de l'action des hormones. Un travail récent [3] propose un aspect nouveau du code de reconnaissance de leurs cibles par les récepteurs nucléaires. En effet, ce travail a révélé la formation d'hétérodimères constitués d'une molécule de $T_{3} R$ et d'une molécule VDR sur des éléments de réponse à la vitamine $\mathrm{D}$ isolés des promoteurs des gènes de calbindine de rat et de souris. Les auteurs montrent que ces éléments de régulation sont constitués de répétition directe du motif consensus séparé par trois ou quatre paires de bases $(\mathrm{pb})$ mais les hexanucléotides qu'ils contiennent sont peu reconnaissables et, de plus, il semble que les $2 \mathrm{pb}$ qui les précèdent soient essentielles : c'est donc dire qu'ils divergent beaucoup du modèle précédent. Quoi qu'il en soit, ces éléments confèrent une réponse hormonale dans leur contexte génique naturel et, fait unique, ils montrent une affinité élevée pour des hétérodimères des récepteurs $T_{3} R$ et VDR, in vitro. De plus, ils conf èrent la double réponse hormonale à un gène rapporteur hétérologue. L'étude de ces cibles et de cibles synthétiques apparentées a montré encore une fois que le demi site aval est celui qui détermine principalement la réponse hormonale. Mais surtout, on a montré par des courbes de réponse hormonale que l'activation du récepteur lié à la moitié aval de l'élément de réponse nécessite une plus faible concentration de ligand que lorsque ce même récepteur est placé en amont. Ces données suggèrent que l'interaction du récepteur avec une séquence spécifique de l'ADN et/ou la position relative de ce site dans un élément de réponse hormonale affecte directement l'interaction entre le récepteur et son ligand, et l'effet transcriptionnel qui en découle. Ces différences de positionnement augmentent jusqu'à dix fois la concentration de ligands néces- saire à l'activation transcriptionnelle. Il en résulte qu'aux concentrations physiologiques, ces éléments de réponse seront sensibles, soit à l'hormone thyroïdienne, soit à la vitamine $\mathrm{D}$, et que ce n'est qu'à des concentrations très élevées de l'autre hormone que le même élément conférera aussi une réponse à la deuxième hormone. Ces observations soulignent l'importance des séquences-cibles dans l'action des récepteurs nucléaires et elles indiquent que le modèle simple basé sur l'espacement entre deux motifs répétés ne suffit pas à définir la spécificité d'action des récepteurs. Il ressort donc du travail de Schräder et al. [2] qu'un même élément de réponse hormonale peut conférer une réponse à plus d'un ligand et que cette dualité résulte de la liaison d'un hétérodimère des récepteurs $T_{3} R$ et VDR à la séquence-cible. Mais, d'une façon plus importante, il montre aussi que la séquence de l'ADN du site d'action peut déterminer la concentration de chacun des ligands hormonaux nécessaires à l'activation transcriptionnelle. Ces travaux suggèrent un nouveau mécanisme par lequel la spécificité d'action hormonale pourrait s'établir sur différents gènes cibles et dans différentes cellules en fonction, non seulement des séquences-cibles, mais aussi du sous-ensemble de récepteurs qui sont présents dans l'une ou l'autre cellule.

J.D.

1. Deutsch J. Ultraspiracle et le récepteur de l'ecdysone ou les relations dans la famille des récepteurs hormonaux nucléaires de la drosophile aux mammifères. médecine/sciences $1993 ; 9$ : 701-5.

2. Lavau C, Jansen J, Weis K. Lamond A, DejeanA. L.eucémie aiguẽ promyélocytaire et acide rétinoïque: le paradoxe. médecine/sciences $1994 ; 10$ 817-24.

3. Schrāder M, Müller KM, Nayeri S, Kahlen JP, Carlberg C. Vitamin $\mathrm{D}_{3}$-thyroid hormone receptor heterodimer polarity directs ligand sensitivity of transactivation. Nature $1994 ; 370: 382-6$. 